\title{
Nuevos PROCESOS, VIEJOS PROBLEMAS. ¿CÓMO SE DEMOCRATIZAN LOS PAÍSES? El CASO de Myanmar
}

\author{
Fernando Pedrosa* \\ Cecilia Noce ${ }^{* *}$
}

\section{Resumen}

El artículo aborda el proceso de democratización de Myanmar tomando como inicio el proceso de independencia nacional, enfatizando los diversos momentos que se vincularon con cambios en el régimen político $y$, sobre todo, con los intentos de avanzar en reformas democráticas. Enfoca su atención en los actores políticos, sus posiciones, cambios e intereses, en especial en el desarrollo del liderazgo de Suu Kyi y las dificultades para consolidarse en el marco de la vigencia de reglas de juego no democráticas. Finalmente, analiza todo este proceso en el marco de los conceptos y modelos propuestos por la literatura de las ciencias sociales para explicar en qué condiciones los países logra avanzar -o no- desde regímenes no democráticos hacia otros híbridos o democráticos.

Palabras clave: democratización, Myanmar, Suu Kyi, transiciones, Asia.

\section{NEW PROCESSES, OLD PROBLEMS. HOW DO COUNTRIES DEMOCRATIZE? THE CASE OF MYANMAR}

\section{Abstract \\ The article will deal with the democra- tization process in Myanmar by taking its}

* Doctor en Procesos políticos contemporáneos. Profesor titular e investigador del Grupo de Estudios de Asia y América Latina, Instituto de Estudios de América Latina y el Caribe, Facultad de Ciencias Sociales, Universidad de Buenos Aires, Buenos Aires (Argentina).ferpedrosa@gmail.com

** Magíster en Análisis cultural y sociología de la cultura. Investigadora del Grupo de Estudios de Asia y América Latina, Instituto de Estudios de América Latina y el Caribe, Facultad de Ciencias Sociales, Universidad de Buenos Aires, Buenos Aires (Argentina). cecilianoce@gmail.com

Recibido: 1 de octubre de 2015 / Modificado: 22 de octubre de 2015 / Aceptado: 5 de noviembre de 2015.

Para citar este artículo

Pedrosa, F. y Noce, C. (2015). Nuevos procesos, viejos problemas. ¿Cómo se democratizan los países? El caso de Myanmar. OPERA, 17, pp. 83-104. DOI: http://dx.doi.org/10.18601/16578651.n17.05 
process of national independence as a starting point. It will also emphasize the various instances in which they were linked to changes in the political regime and especially with the attempts to advance democratic reforms. Focus will be placed on political actors, their positions, changes, and interests, especially the development of Suu Kyi's leadership and the difficulties of consolidating power in a context of the nondemocratic rules of play in force. Finally, an attempt is made to analyze this process in the framework of concepts and models proposed by literature from the Social Sciences in order to explain the conditions in which countries succeed - or fail - to progress from nondemocratic regimes to hybrid or democratic regimes.

Key words: Democratization, Myanmar, Suu Kyi, transitions, Asia.

\section{INTRODUCCIÓN}

El estudio de los procesos de democratización ha integrado constantemente las agendas de las ciencias sociales, en un intento por desentrañar por qué y cómo algunos países transcurren el camino de regímenes no democráticos hacia otros democráticos y viceversa, y a la vez explicar qué condiciones permiten generalizar hipótesis e, incluso, predecir las causas de estos de cambios.

Las preocupaciones de especialistas y académicos sobre los cambios de régimen político vuelven regularmente a los primeros planos cuando algún país aborda uno de estos procesos y, más aún, cuando un conjunto de países lo hace, en el modelo de olas que impuso Huntington (1991). Esto ocurrió recientemente con las llamadas primaveras árabes (y antes con las revoluciones de color) que expandieron los procesos de democratización en zonas donde la tercera ola no había llegado inmediatamente luego del fin de la Guerra Fría. En la actualidad, ocurren procesos de estas características, con avances y retrocesos, como por ejemplo, en Egipto, Túnez y Burkina Faso en África, Fiji en Oceanía o Myanmar y Tailandia en el sudeste de Asia.

Aunque numerosos países de Asia participaron de la llamada tercera ola de las democratizaciones -por ejemplo Filipinas, Corea del sur, Taiwán, Pakistán, Mongolia, Nepal, Tailandia, Bangladesh, Indonesia y, en forma frustrada, Myanmar- estos procesos nacionales y regionales no han sido estudiados con la misma profundidad como los ocurridos en América Latina y Europa.

Sumado a las numerosas discusiones sobre los diferentes tipos de democracias realmente existentes (Collier y Levitsky, 1998), el estudio de un país como Myanmar presenta cuestiones problemáticas ligadas a la especificidad de las tradiciones asiáticas (Lee, 1998) y a la relación con la herencia colonial (Alatas, 2001). Sin embargo, aceptando el riesgo de cierto etnocentrismo, nos parece importante mantener presente una mirada global que permita reflexionar y proponer nuevas posibilidades teóricas para abordar los procesos de democratización contemporáneos.

Este artículo esbozará, en primer lugar, algunos argumentos sobre la importancia del estudio de Myanmar; en segundo lugar, se presentarán muy sucintamente distintos debates teóricos sobre el tema de la democratización 
con la intención de extraer conceptos que ayuden a explicar el rumbo del proceso en el caso elegido; en tercer lugar, se presentará un resumido desarrollo de los hechos que configuran la llamada democratización de Myanmar, ordenando los acontecimientos con una mirada de larga duración que exceda el énfasis en cada movimiento puntual de avance o retroceso.

Finalmente, se hará un análisis a partir de utilizar algunas de las variables teóricas propuestas en la primera parte para analizar su pertinencia en Myanmar y las posibilidades de su inclusión en el universo de los procesos de democratización ya trabajados.

\section{LA IMPORTANCIA DEL PROCESO POLÍTICO DE MYANMAR}

En primer lugar, el estudio del cambio político en Myanmar resulta una oportunidad para continuar investigando casos actuales y, así, incorporar nuevas posibilidades teóricas y empíricas o poner a prueba y corregir teorías ya existentes. Asimismo, la confrontación con nuevos casos sirve para repensar críticamente otros acontecimientos ya ocurridos y sobre los que, posiblemente, tengamos demasiadas certezas, lo cual dificulta el surgimiento de nuevas ideas e hipótesis.

En este sentido, el proceso de Myanmar ha conjugado elementos dispares, que implican desafíos a los modelos teóricos que se detallarán más adelante. De hecho, los sucesos que han marcado la vida política, económica y social del país desde su independencia han implicado simultáneamente tres niveles: la construcción de un régimen político, de un Estado moderno y de una nación.

En segundo lugar, y con argumentos más pragmáticos, Myanmar importa por sus características geográficas, económicas y poblacionales. Ubicado estratégicamente entre China e India, también limita con Bangladesh, Laos y Tailandia; posee una gran costa en el golfo de Bengala, posición que resulta crucial para el comercio de la zona. Su histórica capital fue Rangún (hoy llamada Yangon), hasta que fue reemplazada en 2005 por Naipyidó (o Nay Pyi Taw según la traducción), una ciudad construida a tal efecto ${ }^{1}$; aunque Rangún sigue siendo la ciudad más poblada y el corazón económico del país, el poder político se encuentra en Naipyidó ${ }^{2}$.

\footnotetext{
1 Una medida que recuerda a otra tomada por Pinochet en Chile al trasladar el parlamento a Valparaíso para que los legisladores estuvieran alejados de la capital. Para el caso de Naipyidó se argumentaron razones de seguridad, ya que era más difícil de atacar desde la costa. En algunos medios se afirmó que el Gobierno de George W. Bush había evaluado tal posibilidad en 2003. Sin embargo, la mudanza también tenía que ver con alejar al poder político de las manifestaciones de protesta y el activismo social que en 1988 habían comenzado a aflorar con una intensidad no prevista.

2 El nombre actual-Unión por la República de Myanmar-cambió varias veces desde la independencia. En 1948 pasó a denominarse como República Unión de Birmania, poniendo énfasis en el término "unión" que se relacionaba con la intención de crear un Estado federal. Luego del golpe de 1962, se denominó República Socialista de Unión de Birmana. En 1989, la Junta militar volvió a cambiar el nombre por Unión de Myanmar, y en 2010 se impuso el definitivo Unión por la República de Myanmar. Este último cambio, también incluyó una nueva bandera e himno nacional. Para profundizar ver Houtman (1999).
} 
Además, el país posee recursos naturales inexplorados (gas, diamantes, madera, entre otros) que han atraído la atención de China y Estados Unidos. Como muestra de este interés, Barack Obama, Hillary Clinton, José Manuel Durão Barroso (entonces presidente de la Comisión Europea) y los primeros mandatarios de China y Japón han visitado el país en los últimos años. El presidente de Myanmar ha sido recibido, a su vez, por sus pares de China y Estados Unidos en visitas oficiales. La versión regional del Foro de Davos se reunió en Rangún, y el Banco Mundial otorgó una millonaria línea de crédito para la construcción de un sistema nacional de salud ${ }^{3}$.

Según el último informe del Banco Mundial, Myanmar se encuentra en un periodo de crecimiento económico muy sólido que promedia un $8 \%$ anual desde hace años, al mismo tiempo que la pobreza supera un $35 \%$ de la población, sobre todo en áreas rurales. El crecimiento de la inversión extranjera directa ha resultado crucial y ha permitido, simultáneamente, crear una serie de negocios altamente redituables para empresas extranjeras y comenzar a construir un Estado moderno, lo que se refleja en planes sociales, en un incipiente sistema de retiros y pensiones, y en un desarrollo de las infraestructuras de transporte y comunicación ${ }^{4}$.

En tercer lugar, el país es un caso interesante de aislamiento del entorno interna- cional. Desde 1962 hasta 1989, Myanmar se convirtió en un régimen socialista de partido único, aunque, al mismo tiempo, evitó alinearse claramente con los soviéticos, proponiendo lo que se denominó la "vía birmana al socialismo", que incluía elementos propios como la adopción del budismo como religión oficial (May y Selochan, 2004).

Su aislamiento llama aún más la atención al considerar la coyuntura que vivió la región durante la Guerra Fría: la guerra de Vietnam, los bombardeos norteamericanos en Laos y Camboya y los gobiernos comunistas que allí triunfaron, que hicieron difícil abstraerse del entorno geopolítico (Lagerkvist, 2008).

El aislamiento, que comenzó a abrirse a fines del siglo pasado, se traduce aún hoy en la falta de alineamiento internacional por parte del Gobierno de Myanmar. En años anteriores, China defendió en el ámbito internacional la idea de que Myanmar debía encontrar un camino propio a la democracia y al desarrollo, sin la intervención de las potencias occidentales. La postura china le permitió establecer relaciones económicas privilegiadas, mientras Estados Unidos y la Unión Europea tomaban un camino inverso, aplicando distintos tipos de sanciones.

$\mathrm{Al}$ aumentar significativamente la expansión del capital chino, el Gobierno de Myanmar comenzó a abrirse a capitales de otras regiones (Estados Unidos, Japón, India,

\footnotetext{
3 De 1,6 billones de dólares según un informe de prensa del Banco Mundial. Recuperado de www.worldbank. org

4 World Bank East Asia and Pacific economic update. Adjusting to a Changing World. Recuperado de www.worldbank.org
} 
Tailandia) para contrarrestar una dependencia que ya había generado conflictos entre los militares, y en sectores de la población por los daños ambientales producidos por las empresas chinas (Cerda Dueñas, 2014) 5 .

En cuarto lugar, es necesario mencionar que parte de la importancia del país se debe a la presencia de la líder del mayor partido opositor, la Liga Nacional por la Democracia (NLD por sus siglas en inglés), Aung San Suu Kyi, quien ha logrado un gran predicamento en la opinión pública internacional a pesar de la campańa de desprestigio en su contra organizada desde el Gobierno (Houtman, 1999). De hecho, obtuvo el Premio Nobel de la Paz en 1991 (que no pudo recoger hasta 2012, por sufrir arresto domiciliario) y ha sido el eje de una campaña internacional por la democratización en Myanmar que ha incluido la realización cinematográfica de su historia ${ }^{6}$.

En quinto lugar, en los últimos tiempos, Myanmar ha alcanzado nuevamente la atención de la comunidad y la prensa internacional por la crisis de los migrantes rohingyas, una cuestión, sin embargo, que sucede desde hace décadas. Se trata de una minoría étnica que huye del país perseguida por su religión musulmana y por la extrema pobreza que sufre, como consecuencia de la discriminación política y económica a la que es sometida (Parnini, 2013).

La situación de los grupos étnicos segregados, incluso los más perjudicados y que debieron desplazarse de sus territorios fuera del país, no solo se vincula con condiciones socioeconómicas y las consecuencias imprevistas de desastres naturales, sino con el tema central que trata este artículo: el de las luchas alrededor del régimen político del país y las formas peculiares que adopta este proceso de cambio en un país poblado por diversos grupos étnicos que además conviven en una permanente disputa por porciones de poder ${ }^{7}$.

En síntesis, el futuro geopolítico de Myanmar estimula la presencia de las principales potencias mundiales en el país asiático y el atento seguimiento de su coyuntura política por parte de los especialistas y la prensa internacional.

5 Las relaciones del Gobierno chino con el de Myanmar desmejoraron por la decisión de suspender la ejecución de obras a cargo de empresas chinas. Este malestar se reflejó en la recepción dada a la líder opositora de Myanmar en el Gran Palacio del Pueblo de Beijing que suele utilizarse para recibir a mandatarios extranjeros. Además, el encuentro fue profusamente divulgado por la prensa china. Para profundizar en el tema ver Chenyang y Char (2015).

6 La película del año 2011, cuyo título original fue The Lady (en español "La fuerza del amor") fue dirigida por Luc Besson. Numerosos documentales también abordaron la compleja figura de la líder de la oposición birmana.

7 En Myanmar habitan más de 130 grupos étnicos. El grupo mayoritario (aproximadamente el 60 \%) y quien tiene el control del país son los bamar. Varios de estos grupos se han enfrentado al Estado durante décadas en busca de mayores cuotas de autonomía política y territorial (Lee, 2014). El resultado del censo del 2014, denunciado por "ignorar" a grupos étnicos y por no alcanzar zonas ocupadas por grupos armados, arrojó poco más de 51 millones de habitantes, resultando una cifra menor a la estimada previamente por organismos internacionales. Esta reducción "benefició" al Gobierno ya que aumentó el PBI en un 17\%, entrando al "club" de los países con más de 1000 dólares per capita. Recuperado de http://countryoffice.unfpa.org/ 


\section{DEMOCRATIZACIÓN, DEBATES Y PROPUESTAS TEÓRICAS}

\section{Auge y caída del paradigma de la transición}

El inicio de una serie de cambios de régimen a fines de los años setenta representó la (re)aparición de las democracias en el mapa internacional. Si bien diversas disciplinas abordaron estos procesos, la ciencia política produjo los aportes más significativos, al punto que la "transitología” y "consolidología" (Schmitter y Karl, 1994) se incorporaron como subáreas dentro de la disciplina.

Esto no significa que haya sido el primer enfoque sistemático sobre el tema. Otro grupo importante de análisis se agrupó varias décadas antes en torno a las llamadas teorías de la modernización, que comenzaron a vincular estructuralmente cuestiones ligadas al desarrollo con la capacidad de los países para acceder (o no) a regímenes democráticos al mismo tiempo que cuestionaban la posibilidad de la democratización vinculada a la voluntad de los actores sociales. Con otros métodos y diversos argumentos, los seguidores de las llamadas "teorías de la dependencia", también coincidían con la relación entre dimensiones estructurales y cambios políticos en los países periféricos ${ }^{8}$.

Sin embargo, con la aparición de la tercera ola de la democratización, se planteó una serie de dilemas que estas teorías no podían responder eficientemente. Se debía explicar por qué países tan distintos llegaban en un pequeño lapso temporal a convertirse en democracias más allá de sus distintas condiciones estructurales.

Parecía necesario imponer nuevos puntos de vista, por ejemplo, profundizar en las acciones que desarrollaron los agentes políticos liberados de condicionantes deterministas, enfatizando el papel de la toma de decisiones e interacciones políticas en un contexto de información escasa, ambigua y cambiante (O’Donnell et al., 1994). Así, los nuevos estudios resaltaron la necesidad de contar con los actores nacionales (las élites) que debían tener la capacidad -incluso el talento- necesario para encarar y resolver a su favor las indeterminaciones propias de una transición?

En este sentido, frente a las turbulencias del entorno, la élite debía actuar en forma racional y leal. Incluso, se generalizaba acerca del quiebre de la coalición autoritaria entre "duros" y "blandos" y la importancia de establecer puentes entre estos últimos y los sectores democráticos moderados, siguiendo el ejemplo de Adolfo Suárez en España.

Para estos enfoques teóricos, los pactos (públicos o secretos) conformaron una política esperable por parte de la élite, aunque solo participara un colectivo selecto de actores que, en ese mismo acto, acordaban garantías recíprocas. Esta visión, tomando como modelo la transición española, llevó adelante la idea de

\footnotetext{
8 Para ahondar en estos debates ver Martí (2001).

9 Al mismo tiempo, había un fuerte contenido subjetivo en los deseos de los investigadores por una vuelta al régimen democrático luego de años de dictaduras (Tonelli, 2012).
} 
que diseńos institucionales correctos podían superar los obstáculos de nuevas democracias. Esta dimensión elitista fue criticada por quienes señalaron que así se dejaban de lado a otros actores sociales que también habían sido importantes en el proceso (Vitullo, 2001).

También se remarcó la escasa importancia otorgada por la transitología a las dimensiones internacionales y transnacionales, sobre todo para explicar el origen del cambio y el inicio de la transición (Pedrosa, 2014) ${ }^{10}$. Incluso, algunos autores han señalado que la desaparición del mundo comunista marcó el comienzo de una cuarta ola de democratizaciones, diferenciada de la anterior por el cambio del sistema internacional (McFaull, 2006).

Otra serie de cuestionamientos se dirigieron a la idea de que las transiciones debían tener un resultado lineal, la consolidación democrática o, en cambio, un retroceso autoritario a la vez que cierta "flexibilidad" a la hora de considerar a algunos países como democratizados (Carothers, 2002).

O’Donnell (1996) ha sido clave en el desarrollo de las investigaciones sobre estos temas, ya que estuvo presente en todas las facetas de los debates, incluso abriendo grietas en sus propias posiciones iniciales. Si bien el texto está más pensado para América Latina, la idea de otra institucionalización incorpora la posibilidad de reglas informales en los regímenes políticos tan extendidas en sociedades no del todo occidentalizadas.
Para la región que aborda este artículo, esto se tradujo en la idea de "valores asiáticos", fuertemente impulsados por los exmandatarios de Singapur y Malasia (y sus líderes históricos más importantes), Lee Kuan Yew y Mahathir Mohamad, para argumentar favorablemente por la combinación de tendencias autoritarias en gobiernos electos democráticamente.

La informalidad explicaría la persistencia de la democracia como régimen político, aunque con formas que no son las mismas que las practicadas en los países occidentales. Estas ideas luego se consolidarían en la propuesta de un nuevo tipo de democracia -bautizada como delegativa-, pero también con la definición de un nuevo tipo de régimen político difícil de catalogar con claridad como democracias plenas o bien como autoritarismos puros (Case, 2005). A la vez, esto discutiría la idea central de la transitología sostenida en visiones instrumentales de la democracia y donde las elecciones son el elemento definitorio de este tipo de régimen (Diamond, 2004).

\section{Nuevas visiones sobre la democratización}

Con estos nuevos aportes, la literatura comenzó a alejarse del modelo de las transiciones, ofreciendo explicaciones más amplias y que reflexionaban sobre las condiciones que permitirían la democratización de regímenes autoritarios o el quiebre de regímenes democráticos, sin poner el énfasis en el objetivo final de dicho proceso.

10 Adoptando perspectivas tranéne paron y propuestas teóricas.s que no estasnacionales donde se unen los diferentes espacios políticos/geográficos local-nacional-internacional (Keck y Sikkink, 2000). 
La aparición de conceptos dedicados a regímenes que combinaban aspectos democráticos y no democráticos se puso a la orden del día, sobre todo, para los gobiernos poscomunistas, cuyo desarrollo se mostraba muy distinto al optimismo lineal de los primeros estudios transitológicos.

Levitsky y Way (2010) argumentaron que en lugar de democracias, la tercera ola produjo también "autoritarismos competitivos" como en Malasia, Camboya y Taiwán. Estos regímenes son competitivos porque las elecciones son un medio válido para acceder al poder, pero no pueden considerarse democráticos porque desde el Estado se ponen todo tipo de trabas para que la oposición nunca llegue a reemplazar al oficialismo ${ }^{11}$.

Esto es trascendente en el caso de Myanmar, porque aporta información sobre el peso de la región en la definición de los regímenes políticos nacionales. Las transiciones en Europa del Este mostraron que la distribución de la democracia en el sistema internacional estaba fuertemente influenciada por procesos de difusión entre los Estados, especialmente a nivel regional. Además, no solo los regímenes tenderían a ser similares dentro de las regiones, también hay una fuerte tendencia a que se produzcan transiciones de regímenes de una manera más parecida a la media regional (Gledisch, 2000).

Gleditsch (2002) afirmó que la difusión regional es uno de los principales factores de la democratización, argumentando que los Estados tienden a agruparse regionalmente según el tipo de régimen y que, además, las dinámicas regionales son importantes al dar forma a las perspectivas de la democracia. Weyland (2014) también incorpora la idea de "difusión autoritaria", ya que no solo los ejemplos democráticos son los que se difunden, y esto nuevamente es muy acertado para el sudeste asiático. Levitsky y Way (2010) sostienen que es importante considerar el grado de vinculación con Occidente.

...Las relaciones con Occidente -la influencia cultural y los medios, las redes de élites, los efectos de la manifestación y la presión directa de los gobiernos occidentales- parecen haber aumentado los costos del atrincheramiento autoritario [...] Allí donde las relaciones occidentales eran más débiles, o en donde las hegemonías alternativas no democráticas (como Rusia y China) ejercían una influencia significativa, los regímenes autoritarios competitivos muy probablemente persistían o se movían en una dirección más autoritaria (Levitsky y Way, 2004, p. 171).

La cuestión de la duración de estos procesos, ya señalada por O’Donnell (1996), fue retomada en otro sentido por Weyland (2014) al estudiar comparativamente la relación entre la velocidad y la intensidad de las olas democratizadoras. El autor alemán afirma que la primera ola, originada luego de la Revolución francesa, tuvo una rápida expansión pero un

11 Una característica del caso asiático, es la dificultad para afirmar que los países que la integran hayan transitado hacia algún régimen que pueda catalogarse como democrático, aún luego de la salida de regímenes que efectivamente no lo eran (Funston, 2001). 
retroceso casi inmediato. En cambio, la tercera ola fue más lenta pero su intensidad fue más duradera. Si bien Weyland realiza su trabajo para América Latina y parte de Europa, no deja de ser una hipótesis interesante para aplicar y reflexionar en la coyuntura de Myanmar.

Algunas de las nuevas visiones implicaron un retorno hacia los factores estructurales como variable explicativa. Por ejemplo, Przeworzky y Limongi (1997) volvieron a confirmar empíricamente que existían relaciones entre nivel de desarrollo y tipo de régimen, aunque otros estudios insistieron que esto variaba según las distintas regiones del planeta (Mainwaring y Pérez Lińan, 2004).

Acemoglu y Robinson (2006) postularon que la democratización dependía del conflicto entre ricos y pobres sobre la redistribución. La desigualdad reduciría las probabilidades de democratización porque las élites autoritarias perciben que democratizar en el presente implicaría una suerte de promesa de futuras redistribuciones, con lo cual aumentaría el costo de la democratización. Para el mundo asiático, los autores tomaron como ejemplo de sus investigaciones a Singapur.

En respuesta a estos últimos, y también a Boix (2003) -otro autor que retoma las consecuencias políticas del desarrollo económico y la desigualdad-, Ansell y Samuels (2014) sostienen que la desigualdad también genera presiones para la democratización, sobre todo, a partir de las lecciones del caso brasileño (uno de los países más desiguales del mundo a la hora de democratizarse). Para estos autores, se revertiría entonces la percepción desde el poder: el costo de aceptar la apertura de una transición sería menor que una amenaza revolucionaria $^{12}$.

Ansell y Samuels discuten la unidimensionalidad del concepto de desigualdad de Boix y Acemoglu y Robinson, distinguiendo entre la desigualdad de ingresos y la desigualdad en la propiedad de la tierra, considerando esto como un asunto clave para la comprensión de los patrones históricos de cambio de régimen.

En el contexto asiático, este elemento, vinculado al conflicto social, resulta significativo, por lo extenso de su economía rural y su vinculación con la protesta social como medio privilegiado en la región para abrir procesos de democratización (Del Río Martínez, 2007). En ocasiones, estos movimientos se han vinculado a procesos más amplios de conformación del Estado (Antolínez, et al., 2011) y a las disputas por la construcción de una nación entre diversos grupos étnicos, como en Myanmar.

Otros estudios han reiterado que el accionar de las élites debe tenerse en cuenta más allá de los condicionamientos estructurales, pero al mismo tiempo, sin tomarlos como actores únicos y aislados. Si bien el trabajo de Mainwaring y Pérez Liñan (2009) no se aplica para el caso asiático, proponen una serie de

12 "Es decir, la probabilidad de la democracia está asociada no a cierto nivel de igualdad en el ingreso, sino al incremento de la percepción de desigualdad, que puede advertir a las élites de los riesgos de mantener esa situación de deterioro social, y advertir a los trabajadores de la insostenibilidad de su situación” (Vidal de la Rosa, 2011, p. 2). 
dimensiones combinadas que permiten explicar mejor los procesos de democratización. Pensar la posibilidad de que estas explicaciones "viajen" a Asia no es descabellado, sobre todo, porque este tipo de procesos no parecen poder explicarse desde una sola dimensión.

Según los mencionados autores, las actitudes normativas de los actores con respecto a la democracia y la dictadura tienen impacto en la caída o supervivencia del régimen. También importa el radicalismo o la moderación de los actores en disputa como un elemento que explicaría los cambios o las continuidades. Finalmente, el tipo de régimen predominante en la región es otra variable para tener en cuenta.

Con sus diferencias y similitudes, los estudios precedentes, puestos o no en cuestión, han dejado algunas certezas. Una de ellas es que, más allá de cómo se desarrolle un proceso de cambio de régimen, este siempre estará pleno de tensiones e intereses en disputa y, por tanto, presentará un final incierto donde las estrategias de los actores cuentan de un modo u otro.

La coyuntura de Myanmar es tan actual que en sus distintos análisis se ha privilegiado una lógica de anticipar resultados y, por ello, se ha ido perdiendo un abordaje que intente incluirlo dentro de coordenadas teóricas para ofrecer una explicación de orden más sistemático y que tome una prudente distancia (aunque sin perder de vista) de los acontecimientos.

\section{MYANMAR, UN CASO COMPLEJO EN EL SUDESTE ASIÁTICO}

La vida política contemporánea en Myanmar -antes llamada Unión por Birmania- comienza con el proceso de independencia iniciado en 1947 en el contexto de las descolonizaciones posteriores a la Segunda Guerra Mundial. Como muchos de los países de la zona, la lucha por la independencia despertó un fuerte sentimiento nacionalista que se mezcló con las ideologías propias de la época de la Guerra Fría, y que fue acentuado por la traumática relación con los invasores japoneses que ocuparon el país por varios años y en cuya oposición se fue moldeando la élite política birmana.

Aunque durante el siglo XIX, hubo fuertes enfrentamientos entre birmanos y británicos, la independencia estuvo lejos de los niveles de violencia alcanzados en otros países de Asia y África. El líder birmano y miembro de las Fuerzas Armadas, Aung San, fue quien encabezó este proceso iniciado con el fin de la guerra mundial, al mando de la Liga Antifascista por la Libertad de los Pueblos, que combinaba ideas nacionalistas, budismo y un socialismo democrático poco definido.

Aung San obtuvo una gran victoria en las primeras elecciones parlamentarias como en las constituyentes, y esto le permitió delinear una constitución basada en un Estado federal y parlamentario que concedía autonomía para algunos grupos étnicos minoritarios y que incluso aceptaba, bajo determinadas condiciones, el derecho a la secesión. Como muestra de la ambigüedad entre la influencia británica y las ideas nacionalistas y socialistas predominantes en la élite militar, Birmania rechazó ser parte de la Commonwealth. Al poco tiempo, Aung San fue asesinado y el proceso independentista fue definitivamente concluido por otro militar y político, $\mathrm{U} \mathrm{Nu}$, 
quien firmó en 1948 el acuerdo definitivo con el líder laborista inglés Clement Atlee ${ }^{13}$.

En las elecciones nacionales de 1956, los partidos opositores lograron un fuerte avance, embanderados en diversos reclamos étnicos asociados al territorio y a demandas autonomistas. En 1958, U Nu debió renunciar debido a una coyuntura caracterizada por la combinación de conflictos armados (primero comunistas y luego de carácter étnico), una persistente crisis económica y la debilidad del Gobierno para enfrentar ambas problemáticas.

Frente a esta situación que parecía amenazar la integridad territorial de Birmania, los militares, en la persona de Ne Win, ocuparon el Gobierno legalmente, por pedido del parlamento, entre 1958 y 1960. El ejército birmano tenía un fuerte prestigio producto de su lucha anticolonial y, al mismo tiempo, estaba relacionado con la conducción de Estado desde su conformación. Pero fue en esta participación directa en 1958-1960 que los militares se vincularon directamente con la experiencia de gestión de los asuntos públicos y se sintieron capaces de reemplazar a los políticos (Gomá, 2014).

En 1960, U Nu ganó nuevamente las elecciones y ocupó el cargo de primer ministro, sostenido en la promesa de transformar el budismo en la religión oficial del Estado. Su triunfo fue contundente sobre un partido apoyado por el mismo ejército. La población birmana era y es mayoritariamente budista, lo cual fue importante para lograr apoyos electorales pero, a la vez, produjo mayor tensión con los grupos minoritarios que ya estaban alzados en armas.

$\mathrm{Al}$ mismo tiempo, las ideas socialistas en la élite birmana comenzaron a radicalizarse, sobre todo entre los cuadros militares, lo que sería de gran importancia para el futuro. El Gobierno de U Nu mantenía un estilo cercano a la socialdemocracia, con una intervención estatal muy intensa pero abierta a la participación privada, con vigencia de un régimen parlamentario multipartidista y relaciones con el exterior ${ }^{14}$.

Los militares tenían otras ideas y, en 1962, tomaron el poder por medio de un golpe de Estado que devolvió el Gobierno a Ne Win, quien acusó a $\mathrm{U} \mathrm{Nu}$ de permitir la influencia extranjera en el país y de ser incapaz de evitar la amenaza de disgregación territorial. Ne Win suspendió la Constitución de 1947, creó un partido único (el Partido del Programa Socialista de Birmania, pPsB) y en busca de la "vía birmana al socialismo", se radicalizó en gran parte influido por los acontecimientos que ocurrían al mismo tiempo en China y Vietnam. El pPSB se construyó al estilo de los partidos comunistas al punto que durante años solo podían integrarlo los hombres de mayor confianza del régimen, generalmente militares (Gomá, 2014).

\footnotetext{
13 El sistema parlamentario poseía una Cámara de Diputados y otra de de Nacionalidades, un primer ministro (el mencionado $\mathrm{U} \mathrm{Nu}$ ) y un presidente (Shwe Thaik).

14 En 1961, U Thant, exfuncionario y amigo de U Nu, fue designado secretario general de la onU y el primer asiático en ocupar ese cargo. En la gestión del birmano se desempeñaba la hija del asesinado líder Aung San, Aung San Suu Kyi. Sus relaciones con el Gobierno birmano surgido del golpe de Estado no fueron nada buenas.
} 
Sin embargo, con el correr de los años, más que con una dirección unipersonal, el poder pasó a ser compartido y grupal dentro de las Fuerzas Armadas. Los militares se hicieron cargo de la totalidad de la administración del país forzando la nacionalización de la propiedad privada, la planificación centralizada de la economía, como también el fin de toda autonomía para los grupos minoritarios.

Este último fue uno de los temas centrales en la agenda militar. Una de las prioridades del golpe fue hegemonizar étnicamente el ejército que desde tiempos de Aung San se había conformado federalmente y a partir de acuerdos interétnicos; de hecho, un oficial de la minoría Karen fue nombrado como primer jefe del ejército de Birmania.

Desde el punto de vista económico, $\mathrm{Ne}$ Win intentó implementar un proceso de industrialización acelerada que, además de fracasar, perjudicó las posibilidades de supervivencia que otorgaba a la población la producción agraria tradicional. Los intentos de generar cambios estructurales en forma rápida y autoritaria no fueron originales (en China se presentaron desde "el gran salto adelante" hasta la "revolución cultural"), y sería poco después, en Camboya, donde se llevaron hasta sus últimas consecuencias bajo la dictadura de Pol Pot (Kiernan, 2010).

El comercio exterior y el turismo fueron prohibidos, por lo que indios, chinos (tradicionalmente vinculados al comercio), así como empresas extranjeras se retiraron del país. De hecho, se iniciaba el periodo caracterizado por el aislamiento absoluto de Birmania, tanto de las potencias occidentales capitalistas como de los países comunistas.

A pesar del aislamiento, la combinación de crisis interna y los problemas globales que caracterizaron los inicios de los ańos setenta, golpearon también a Birmania generando descontento entre la población. El Gobierno militar apeló para eso a su arma más utilizada, la represión, aunque resultó insuficiente para aplacar la situación. Así, desde la cima del poder, comenzó a darse más importancia al partido y a imponer a este como centro del poder en reemplazo del Consejo revolucionario militar, en conjunto con un creciente discurso ligado al nacionalismo budista.

El régimen pasaba así de sostenerse en un partido de cuadros a uno de masas, en la búsqueda de nuevas fuentes de legitimidad (Gomá, 2014). La transformación le permitió prolongar más de una década su estancia en la cúspide del poder. Los cambios se vieron plasmados en la nueva Constitución que entró en vigencia en 1974. El país cambió su nombre a República Socialista de la Unión de Birmania y el pPsB se convirtió en el órgano de poder más importante, reconocido como tal por las mismas leyes del país. La tarea de consolidación del partido como órgano máximo del Estado pareció coronarse en 1981, cuando Ne Win renunció a la presidencia y continuó siendo el hombre fuerte del país como jefe del partido ${ }^{15}$.

15 Al estilo de la uRss, donde la autoridad máxima del país no era el presidente sino el secretario general del Partido Comunista. 
Sin embargo, Myanmar no pudo abstraerse de la crisis de la época, como tampoco de la influencia de la ola democratizadora que llegaría a la región a finales de la década de los ochenta. El 8 de agosto de 1988, la ciudad de Rangún fue escenario de una serie de protestas conocidas como el levantamiento 8888. Si bien estudiantes y monjes fueron reprimidos de manera brutal, las consecuencias políticas tanto hacia dentro del régimen como hacia afuera fueron de suma importancia, gestándose un nuevo golpe de Estado en 1990, para recomponer el orden impugnado.

Para ello se conformó el Consejo para la Restauración del Orden y la Ley del Estado (sLORC por sus siglas en inglés) ${ }^{16}$ y la instauración de la junta militar con el general Saw Maung como máxima autoridad. Esto marcó el principio del fin de la presencia dominante de Ne Win, aunque su influencia se mantuvo en las sombras por varios años más, hasta su arresto en el año 2001, acusado de apoyar un intento de golpe de Estado.

En el plano exterior, la represión y el golpe produjeron una fuerte reacción internacional que se tradujo en sanciones económicas y en el bloqueo a la ayuda exterior por parte de Estados Unidos y la Unión Europea. Consciente de su debilidad, el nuevo Gobierno inició un proceso de apertura caracterizado por la legalización del comercio internacional, la lenta desregulación de los mercados y el establecimiento de relaciones comerciales con los países de la región que no estaban de acuerdo con las sanciones impuestas por Estados Unidos y la Unión Europea, principalmente Tailandia, China y Japón. Un elemento clave del proceso fue el ingreso del país a la ASEAN en 1997.

En materia política, se convocó a elecciones generales para la conformación de una Asamblea Constituyente en 1990, las primeras realmente libres desde el golpe de Estado de 1962. La fuerte movilización de monjes y estudiantes había dado lugar a la creación de casi un centenar de partidos y grupos políticos que estaban expectantes y movilizados por los resultados electorales.

A pesar del control llevado a cabo por el Gobierno militar y su confianza en el éxito de la liberalización del régimen, las elecciones resultaron en una contundente victoria del NLD de Aung San Suu Kyi, superando el 70\% de los votos. La Junta militar, sin embargo, se negó a reconocer los resultados y condenó a arresto domiciliario a numerosos activistas y dirigentes políticos, entre ellos a la líder opositora, quien, como ya se indicó, recibió el apoyo internacional y posteriormente el Premio Nobel de la Paz.

En 2003, en el marco del relajamiento del arresto domiciliario, Aun San Suu Kyi, junto a una comitiva de la NLD que realizaba actividades de campaña, fue atacada por partidarios oficialistas en una acción que arrojó gran cantidad de muertos y heridos. El hecho produjo la condena mundial, y el entonces presidente Clinton y la Unión Europea endurecieron

16 En 1997, fue reemplazado por el Consejo de Estado para la Paz y el desarrollo (sPDC). 
las sanciones al país asiático ${ }^{17}$. La presión internacional, y la deslegitimación interna del régimen, produjeron que el entonces primer ministro Khin Nyut presentara una "Hoja de ruta hacia una democracia disciplinada", que incluía una nueva Constitución y el llamado a nuevas elecciones.

Sin embargo, la persistencia de las dificultades económicas mantuvo la crisis de 1988 en estado de latencia. De hecho, en 2007 hubo aumentos del combustible de hasta el $500 \%$, y eso volvió a colocar en la superficie el conflicto social, sobre todo con el colectivo de los monjes (shangá) quienes son un sector muy poderoso en un país mayoritariamente budista.

Las constantes movilizaciones de los monjes por el país representaban un desafío al Gobierno, que reprimió sin prever que eso aumentaría el impacto de la protesta, conocida internacionalmente como la revolución azafrán, por el color del atuendo de los monjes ${ }^{18}$. El impacto de la protesta de monjes, estudiantes y activistas en la ciudad de Rangún (cuyo nombre había cambiado junto al del país por Yangon), produjo un significativo cambio en las políticas de no intervención del Gobierno chino, quien solicitó a su par de Myanmar moderación en la resolución del conflicto ${ }^{19}$.
Como consecuencia del malestar generalizado, el Gobierno aceleró el proceso electoral aunque la aprobación de la Constitución en 2008 incorporó mecanismos que reforzaron el poder militar. Así, se previó un cupo fijo del $25 \%$ de los escaños para los militares en la Asamblea Nacional y en las regionales que, combinado con un alto umbral de votos parlamentarios para reformar la constitución, impide cualquier cambio sin el apoyo de las Fuerzas Armadas (De Miguel Calabria, 2011).

$\mathrm{Al}$ mismo tiempo, se estableció la elección presidencial indirecta, debiendo los parlamentarios elegir entre una terna que en la práctica debe contar con el visto bueno del poder militar. Incluso, los ministerios relacionados con la seguridad dependen directamente de las Fuerzas Armadas. Pero los artículos más criticados de la Constitución son los que regulan el funcionamiento de los partidos políticos; entre ellos, la Sección 59 (f) que establece que los candidatos a presidente, sus padres, esposo/a, hijos o los esposos de los hijos no deben ser ciudadanos extranjeros. La prohibición estaba indudablemente dirigida a impedir la postulación de Suu Kyi, que estaba casada con un inglés y cuyos hijos eran de la misma nacionalidad.

\footnotetext{
17 "La Asociación de Naciones del Sudeste Asiático (ASEAN) rompió su tradicional silencio, admitiendo incluso que dicho evento tenía un impacto negativo en el organismo regional. El incidente propició un intenso y ríspido debate al interior de ASEAN, destacando la postura del entonces primer ministro malasio, Mahatir Mohamed, quien propuso la expulsión de Myanmar en tanto no fuera liberada Suu Kyi” (Cerdas Dueńas, 2014, p. 23).

18 Como la Revolución de las rosas en Georgia (2003), la naranja en Ucrania (2004), la de los tulipanes en Kirguistán (2005), la del cedro en el Líbano (2005), la de los jazmines en Túnez (2010), entre otras.

19 En 2008, la situación empeoró con la llegada del huracán Narguis que produjo más de 100.000 muertos y 800.000 desplazados. El Gobierno de Myanmar se opuso a la ayuda internacional y eso perjudicó aún más a las regiones donde habitaban minorías étnicas (Urech, 2012).
} 
La legislación de 2010 para la regulación de los partidos políticos aumentó las restricciones al prohibir la participación política a presos sentenciados, religiosos (a quienes además se les prohibía votar), funcionarios, extranjeros y grupos ilegales. Afectaba, asimismo, a la diáspora birmana ya que cualquier dirigente político refugiado en el extranjero quedaba inhabilitado de participar. Por otro lado, los partidos no podían recibir ayuda o apoyo de países extranjeros, ni podían producir discursos que alentaran sentimientos religiosos o étnicos.

Las primeras elecciones regidas por este marco legal se llevaron a cabo el 7 de noviembre de 2010, para la constitución del Parlamento. El NLD no participó de la elección y así el oficialista Union Solidarity and Development Party (USDP) tuvo el camino allanado, logrando 259 bancas de las 326 puestas en juego (De Miguel Calabria, 2011). Producto de estas elecciones se eligió de la terna de candidatos al general Thein Sein, desde entonces el hombre fuerte del régimen.

Sin embargo, al momento de asumir el cargo, el nuevo presidente realizó un anuncio de apertura que sorprendió a propios y extraños. Desde entonces, se aceleró un proceso de liberación de prisioneros políticos, y una ampliación de la libertad de participación y expresión.

Asimismo, Thein Sein realizó modificaciones al interior del poder militar que implicaron cambios en el gabinete, entre ellos, el reemplazo del vicepresidente, Tin Aung Myint Oo, representante del ala dura del Ejército, por otro reformista. Esto le permitió profundizar reformas sobre las empresas militares que ejercían un rol poderoso en la economía del país (Callahan, 2012).

Las elecciones complementarias del 2012 resultaron importantes, a pesar de la escasa cantidad de escańos en juego, ya que constituyeron una prueba de la voluntad de apertura del Gobierno de Thein Sein ${ }^{20}$. Además, le permitieron a Suu Kyi lograr una banca parlamentaria y, así, legitimar con su presencia este renovado intento de apertura política.

Otro de los políticos reformistas en conseguir un lugar importante luego de las elecciones de 2010 fue el general Thura Shwe Man que, como speaker de la cámara baja, ha cumplido un rol político crucial $^{21}$. La relación negociada entre Suu Kyi y Shwe Man se evidenció en el hecho de que la líder opositora haya sido nombrada como presidenta de la Comisión parlamentaria para el Estado de Derecho y la Paz dedicada, entre otros asun-

20 Eran 45 bancas vacantes (sobre 498 elegibles para ambas cámaras) porque miembros del partido oficial asumieron funciones ejecutivas.

21 Probablemente jugará un papel importante en el futuro como consecuencia de su intención de construir un propio proyecto político más allá de la estructura oficialista, aunque por eso mismo fue destituido de la presidencia del usDp, en medio de fuertes rumores de desavenencias internas con el presidente Thein Sein. Myanmar's Electoral Landscape, Asia Report 266, 28 de abril 2015. 
tos, a debatir sobre las problemáticas étnicas del país ${ }^{22}$.

Además, la Comisión electoral, a partir del ingreso de diferentes fuerzas políticas, ha llevado a cabo varias modificaciones que probablemente tengan un impacto positivo en las elecciones de 2015. Entre ellas, la apertura de los registros de los partidos políticos, la disminución de los costos para presentarse a elecciones, la reforma y adecuación del voto por adelantado y la aceptación de veedores internacionales.

Como resultado de las reformas encaradas, Estados Unidos y la Unión Europea han levantado las sanciones económicas que pesaban sobre el país, como parte de una nueva política hacia Myanmar que comenzó a poner en juego la geopolítica regional y la contención de China como elemento preponderante.

Uno de los aspectos que genera mayor controversia y a la vez expectativas del proceso de transformación es la relación con las minorías étnicas. Por un lado, el Gobierno puede contar, entre sus mayores logros del periodo, la firma de los primeros ceses de fuego con los brazos armados de diferentes grupos étnicos, que concluyó en la firma de un acuerdo nacional el 15 octubre 2015. Sin embargo, varios grupos importantes han quedado relegados e, incluso, en los últimos días de negociación continuaban los combates en diferentes zonas del territorio ${ }^{23}$.

El proceso de pacificación resulta crucial para el futuro del país porque la amenaza de desintegración territorial ha sido siempre una excusa para legitimar el rol activo de las Fuerzas Armadas. Además, los grupos étnicos están situados en zonas ricas en recursos naturales que permanecen sin ser explotados.

Un punto sensible de las políticas llevadas adelante, es la dramática situación económica, social y política de la minoría rohingya, que genera presiones internacionales y regionales tanto para el gobierno como para la oposición.

Las elecciones de noviembre 2015 que transcurrieron de forma libre y transparente, con la excepción de la restricción impuesta a los ciudadanos musulmanes, significaron un rotundo triunfo para la NLD que se alzó con un $70 \%$ de los escańos en juego ${ }^{24}$.

La transición hasta la formación de un nuevo gobierno sigue planteando un final abierto. Será un momento clave para desentranar el futuro del proceso político de Myanmar ya que el oficialismo deberá negociar internamente al próximo candidato, en un contexto de derrota, y allí se jugará el destino de la corriente reformista. Sobre todo, si se pusiera en duda su continuidad al frente del Estado.

\footnotetext{
22 En esta función Suu Kyi fue criticada por sus posiciones ambiguas -o el silencio- sobre la situación de la minoría Rohingya. Incluso entre los 1.000 candidatos el NLD para las elecciones de noviembre de 2015 ni uno solo es musulmán. En el oficialismo, a la inversa, y como mencionáramos anteriormente, se dejó fuera de combate a Shwe Man, al que le fue aplicada la misma ley proscriptiva que a Suu Kyi.

23 “Myanmar Times: A fissure among ethnic armed groups”. Recuperado de http://www.burmanet.org/

24 The Guardian, "Final Myanmar results show Aung San Suu Kyi's party won 77\% of seats". Recuperado de http:// www.theguardian.com/s
} 
Para la oposición tampoco será fácil. Aún en el triunfo, la imposibilidad de nominar a Suu Kyi pondrá al partido frente a la necesidad de generar nuevos liderazgos o buscar alguna figura de consenso que pueda presentarse como candidato entre los sectores reformistas del oficialismo. En este marco, la figura del actual presidente, quien ha decidido postularse a pesar de haber rechazado anteriormente esa posibilidad, resultará fundamental para el destino del proceso que él mismo abrió casi cinco años atrás.

\section{La democratización de Myanmar, conclusiones tentativas}

¿Cómo pensar la coyuntura de Myanmar más allá de describir los movimientos de los actores en el sistema político? Si bien la transitología clásica no parece el marco teórico adecuado para abordar el proceso descrito sumariamente, se pueden rescatar algunas cuestiones señaladas en estos estudios y que pueden ser de utilidad para detectar el rumbo de las acciones políticas de los diferentes actores.

Por ejemplo, la existencia de una división de la coalición oficialista entre duros y blandos, a la que la transitología le otorga un lugar clave a la hora de explicar la transición a una democracia. En la práctica, esta ruptura en el seno del oficialismo, permitió el ingreso del NLD a la vida parlamentaria. La cuestión de los pactos (públicos o no) también es un elemento clave en esta tradición de investigaciones sobre la democratización.

En este sentido, en Myanmar se puede observar una renovada tendencia al diálogo y al acuerdo entre los actores, dinamizada, además, por la vida parlamentaria cotidiana, que ha consolidado las redes de relaciones personales más allá de los respectivos espacios políticos de pertenencia (Egreteau, 2014) y que incluso resultaron en un encuentro personal entre el presidente Thein Sein y la líder de la oposición Suu Kyi.

En este marco es que también pueden leerse las reformas a la legislación electoral de 2010, que permitieron una participación más amplia en las elecciones complementarias de 2012. Como se señaló, las modificaciones han sido el resultado de las negociaciones y los consensos dirimidos en la Comisión electoral que fue integrada con representantes de diversas organizaciones tanto oficialistas como opositoras.

Las dimensiones propuestas por Mainwaring y Pérez Liñan para América Latina son interesantes al aplicarlas a Myanmar, sobre todo, por la renovada atención puesta en los actores políticos. Sin duda, la política de Myanmar se construye en las decisiones estratégicas de los actores, aun sin minusvalorar los condicionamientos estructurales a los que se someten, producto de coyunturas económicas globales o rasgos sistémicos.

La protesta social ha sido considerada un elemento característico de las democratizaciones asiáticas, y en Myanmar jugó un rol similar al del resto de los países de la región, marcando límites en momentos precisos, pero luego, dejando lugar a las dinámicas propias de la política. Tanto las protestas del 8888, como la revolución del azafrán produjeron cambios que, aunque lentos, se han revelado difíciles de revertir. De hecho, la continua expansión 
de las organizaciones partidarias ha abierto instancias de participación formal que no existían en los movimientos 8888 .

A la vez, las estrategias gubernamentales -combinando represión y cooptación- restringieron mucho el accionar de grupos inorgánicos o extremistas y la política ahora parece un juego aceptado por las élites, incluso las de los grupos étnicos minoritarios. Este elemento permite abrigar moderadas expectativas sobre una mayor competitividad futura en el escenario electoral.

Retomando las variables propuestas por Mainwaring y Pérez Liñan, las preferencias normativas de los actores por la democracia (oficialistas y opositores) no parecen ser muy profundas, lo cual aporta algunas dudas sobre el rumbo general del proceso en un sentido contrario al del párrafo anterior. Sin embargo, la moderación de la oposición, en especial de la líder del NLD, y una renovada relación de Occidente con el Gobierno de Myanmar, permitiría argumentar que el rumbo del régimen se encuentra abierto y en manos de las élites políticas del país asiático.

La influencia regional e internacional como dimensión que afecta el rumbo del régimen político parece ser aplicable en los últimos años en Myanmar. En el plano internacional, las sanciones y el aislamiento llevaron al país a priorizar los vínculos con China, que no demandó ningún cambió significativo en esa esfera. Retomando a Levitsky y Way, la ausencia de fuertes tradiciones y vínculos con el mundo occidental (asociado a la exis- tencia de un partido/Estado burocrático y consolidado) también permiten pronosticar que la posibilidad de una democracia liberal y republicana no es una opción realista sin descartar la conformación de un régimen autoritario pero electoralmente competitivo.

Tomando como base a la región, tampoco existe ningún resquicio para esperar que Myanmar recorra un camino muy diferente al de sus vecinos. Según Freedom House, ningún país del sudeste asiático es considerado libre ${ }^{25}$. En este aspecto mencionado por Gledisch, si Myanmar transitara hacia un régimen más cercano a la media regional, sería algún tipo de régimen híbrido, donde aún no queda claro el grado de competitividad de las elecciones.

Con respecto a considerar una relación directa entre variables como desarrollo, desigualdad y el tipo de régimen, en Myanmar se encuentra un primer escollo de forma y es que aún no existen cifras para hacer experimentaciones con cierto grado de credibilidad estadística. Sin embargo, el crecimiento de la economía y la presencia creciente del capital extranjero generan desafíos de suma importancia que pueden analizarse desde la perspectiva de Acemoglu y Robinson, en el sentido de que la democratización presente se relaciona con la posibilidad de una redistribución futura.

Al mismo tiempo que las élites disputan por el poder político, por redefiniciones étnicas y proyectos personales, la cuestión de los clanes enfrentados por su vinculación con la corrupción (sobre todo en el oficialismo) es un elemento que no puede desdeñarse y que

25 Recuperado de https://freedomhouse.org/program/southeast-asia-human-rights 
dificulta el avance en la institucionalidad formal de la democratización.

No obstante, un fuerte elemento estabilizador para al paso a un régimen más abierto es la necesidad de mantener un "clima de negocios" previsible y aceptable para las inversiones extranjeras, en lo que se ha convertido en un objetivo común para todos los actores políticos del país que tienen algo para ganar con un escenario de vertiginoso crecimiento económico.

Otro aspecto de dicho objetivo, es la necesidad de pacificación del territorio. Una de las cuestiones centrales que han explicado y, sobre todo, justificado la larga intervención militar son los desafíos a la integridad territorial del país. Una de las intervenciones del Gobierno más activas fue para acordar el fin de conflictos armados con los grupos étnicos, en lo que se denominó "capitalismo de alto el fuego", por su vinculación con la necesidad de tranquilizar zonas susceptibles de recibir inversiones.

La paz, por tanto, resulta crucial para el futuro político y económico del país, ya que los territorios sobre los cuales los diversos grupos étnicos reclaman su autonomía, son a la vez aquellos de mayores riquezas.

Uno de los temas más delicados está relacionado con las políticas del Estado con respecto a la minoría musulmana, llegando incluso a no reconocerle derechos políticos a partir de la imposibilidad de registrarse como candidatos y votantes.

El consenso que existe sobre estas políticas aúna a miembros del oficialismo y de la oposición enmarcados en un creciente "nacionalismo budista". La radicalización del fenómeno y la inacción del Gobierno abren serias dudas sobre la capacidad del Estado para resolver esta problemática a corto plazo.

Retomando la idea de Weyland sobre la relación entre intensidad y duración, existe cierta confianza en el poder militar en la institucionalización de los objetivos de las Fuerzas Armadas, elaborados con éxitos y fracasos desde 1962. Incluso si debieran abandonar la responsabilidad directa sobre la gestión cotidiana de partes importantes del Estado.

El proceso de Myanmar, por un lado, recuerda en algunos aspectos al chileno y a las características de una democratización "desde arriba", con una fuerte herencia institucional que condiciona a los actores civiles, a la vez que crea espacios de acción que resultan importantes. La participación del NLD en las elecciones ha supuesto una aceptación implícita de las reglas de juego institucionales, aun en su búsqueda de transformarlas en un futuro cercano.

Por otro lado, existen algunas características específicas que señalan la originalidad del proceso de democratización de Myanmar, especialmente, el hecho de que implique tres procesos simultáneos: la construcción de un régimen político, de un Estado y de una nación.

Aunque esta simultaneidad tendría alguna semejanza con la ocurrida en los países poscomunistas europeos, donde el cambio de régimen coincidió con redefiniciones nacionales y étnicas, o con las democratizaciones actuales de Egipto y Túnez, la diferencia radica en que Myanmar también está construyendo un Estado desde lo burocrático, partiendo desde el inicio de este tipo de proceso, es decir, en la lucha por el control del territorio y la monopolización de la violencia. 
La complejidad del proceso que vive Myanmar debe entonces multiplicarse por tres (régimen político, Estado y nación) y los hechos vinculados al desarrollo, a los conflictos étnicos, a la lucha política y a los factores internacionales quedan necesariamente atravesados por las acciones de las élites políticas en el marco de las múltiples decisiones e intereses simultáneos que deben afrontar.

Aunque parece poco probable un retroceso a los días previos a la asunción de Thein Sein, las complejas relaciones entre las Fuerzas Armadas y el Gobierno, así como el papel de los oficiales parlamentarios y las normas constitucionales proscriptivas o destinadas a proteger el poder militar, son cuestiones que generan dudas sobre el futuro del país y que encuentran en el test electoral de noviembre de 2015, la vara que marcará el destino del régimen birmano.

\section{REFERENCIAS}

Acemoglu, D. y Robinson, J. (2006). The Economic Origins of Dictatorship and Democracy. Cambridge: Cambridge University Press.

Alatas, S. F. (2001). Alternative Discourses in Southeast Asia. Sari, 19, 49-67.

Antolínez, J., Delgado, Hernández, A., García, C., Sierra, C., Felipe, A. y Támara, P. (2011). Conformación de Estados en el sudeste asiático: una deconstrucción de los estudios de área. Oasis, 16, 83-119.

Ansell, B. W. y Samuels, D. (2014). Inequality and Democratization: An Elite-Competition Approach. Cambridge: Cambridge University Press.

Borx, C. (2003). Democracy and redistribution. Cambride: Cambridge Univ Press.
Callahan, M. (2012). The generals loosen their grip. Journal of Democracy, 23 (4), 120-131.

Carothers, T. (2002). The End of the Transition Paradigm. Journal of Democracy, 13 (1), 5-21.

Case, W. (2005). Southeast Asia's hybrid regimes: When do voters change them? Journal of East Asian Studies 5 (2), 215-37.

Chenyang L. y Char, J. (2015). China-Myanmar relations since Naypyidaw's political transition: How Beijing can balance short-term interests and longterm values. Singapore: Rajaratnam School of International Studies.

Cerda Dueñas, C. (2014). Coyunturas y recelos de la reinserción internacional de Myanmar. México y la Cuenca del Pacifico, 9, 19-46.

Collier, D. y Levitsky, S. (1998). Democracia con adjetivos. Innovación conceptual en la investigación comparativa. Revista Ágora, 8, 99-122.

De Miguel Calabria, E. (2011). ¿Hacia dónde se encamina la transición birmana? En UNISCI Discussion Papers, 26. España: Universidad Complutense de Madrid.

Del Río Martínez, A. (2007). La tercera ola democrática en Asia: explicación política para un fenómeno político. En Actas del XII Congreso Internacional de ALADAA. México: Benemérita Universidad de Puebla.

Diamond, L. (2004). Elecciones sin democracia. A propósito de los regímenes híbridos. Estudios Politicos, 24, 117-134.

Egreteau, R. (2014). Legislators in Myanmar's First "Post-Junta" National Parliament (2010-2015): A Sociological Analysis. Journal of Current Southeast Asian Affairs, 33 (2), 91-124.

Funston, J. (ed.) (2001). Government and Politics in Southeast Asia. Singapore: Institute of Southeast Asian Studies. 
Gledisch, K. (2000). International dimensions of democratization. Copenhague: ECPR Joint Session Meeting.

Gleditsch, K. (2002). All International Politics is Local - The Diffusion of Conflict, Integration, and Democratization. Ann Arbor: The University of Michigan Press.

GomÁ, D. (2014). Socialismo y ejército: la "vía birmana al socialismo" y la consolidación del partido del programa socialista de Birmania (1962-1974). Historia y Política (30), 279-303.

Houtman, G. (1999). Mental Culture in Burmese Crisis Politics: Aung San Suu Kyi and the National League for Democracy. Tokyo: Tokyo University of Foreign Studies.

Huntington, S. (1991). The Third Wave: Democratization in the late 20th Century. Norman: University of Oklahoma Press.

Keck, M. y SikKink, K. (2000). Activistas sin fronteras. México: Siglo xxI.

Kiernan, B. (2010). El régimen de Pol Pot. Raza, poder y genocidio en Camboya bajo el régimen de los jemeres rojos, 1975-1979. Prometeo: Buenos Aires.

LAGERKVIST, J. (ed.) (2008). Between Isolation and Internationalization: The State of Burma. Hallvigs Reklam: The Swedish Institute of International Affairs.

LeE, E. J. (1998). ¿Valores asiáticos como ideal de civilización? Nueva Sociedad (155), 111-125.

LeE, J. (2014). Explaining Myanmar's regime transition: the periphery is central. Democratization, 21 (5), 780-802.

LEVITSKY, S. y WAY, L. (2004). El surgimiento del autoritarismo competitivo. Elecciones sin democracia. Estudios Politicos (24), 159-176.

Levitsky, S. y WaY, L. (2010). Competitive Authoritarianism: Hybrid Regimes After the Cold War. New York: Cambridge University Press.
Mainwaring, S. y Pérez-Liñán, A. (2004). Nivel de desarrollo y democracia: el excepcionalismo latinoamericano (1945-1996). América Latina Hoy (36), 189-248.

McFaull, M. (2006). The missing variable: The "International system" as the link between third and fourth wave models of democratization. Working Papers, 58. Stanford: CDDRL.

Mainwaring, S. y Pérez-Liñan, A. (2009). ¿Por qué son importantes las regiones? Especificidades regionales y difusión de la democracia a escala regional. Revista SAAP, Argentina, 3 (3), 523-566.

May, R. y Selochan, V. (2004). The Military and Democracy in Asia and the Pacific. ANU E Press. Martí I Puig, S. (2001). ¿Promesas incumplidas? Un balance crítico de las teorías del cambio político y su aplicabilidad en América. Revista CIDOB d'Afers Internacionals, (54), 113-137.

Przeworski, A.; Álvarez, M. E.; Cheibub, J. A. y Limongi, F. (2000). Democracy and Development. Political Institutions and Well-Being in the World, 1950-1990. New York: Cambridge University Press.

O’Donnell, G. (1996). Otra Institucionalización. Revista Ágora, 5, 5-28.

O’Donnell, G., Schmitter, P. y Whitehead, L. (1994). Transiciones desde un gobierno autoritario. Barcelona: Paidós.

Parnini, S. N. (2013). The Crisis of the Rohingya as a Muslim Minority in Myanmar and Bilateral Relations with Bangladesh. Journal of Muslim Minority Affairs, 33 (2), 281-297.

Pedrosa, F. (2014). La influencia en las transiciones de la "tercera ola" en América Latina. Debates, definiciones y propuestas teóricas. PolHis. Boletín Bibliográfico Electrónico del Programa Buenos Aires de Historia Politica, 12, 208-224. 
Przeworzky, A. y Limongi, F. (1997). Modernization theories and facts. World Politics, 49 (2), 155-183.

Schmitter P. y Karl, T. L. (1994). The Conceptual Travels of Transitologists and Consolidologists: How Far to the East Should They Attempt to Go? Slavic Review. 53 (1), 173-185.

Tonelli, L. (2012). Guillermo O’Donnell y su compromiso con la democratización. Revista Temas y Debates (24), 105-114.

UreCH, P. (2012). Myanmar/Birmania, análisis de un conflicto. Boletín del Instituto de Estudios sobre Conflictos y Acción Humanitaria (IECAH).
Vidal de la Rosa, G. (2011). Equidad y democracia: Comentarios teórico-metodológicos. Argumentos, 24 (65), 109-133.

Vitullo, G. E. (2001). Transitologia, consolidologia e democracia na América Latina: uma revisão crítica. Revista de Sociologia e Política, (17), 53-60.

Weyland, K. (2014). Making Waves: Democratic Contention in Europe and Latin America since the Revolutions of 1848. Cambridge: Cambridge University Press. 